REVISTA MATEMÁTICA COMPLUTENSE

Volumen 12, número 1: 1999

http://dx.doi.org/10.5209/rev_REMA.1999.v12.n1.17199

\title{
Curves in $P^{2}\left(\mathbb{C}^{\prime}\right)$ with 1-dimensional symmetry.
}

\author{
A.A. du PLESSIS and C.T.C. WALL
}

\begin{abstract}
In a previous paper we showed that the existence of a 1parameter symmetry group of a hypersurface $X$ in projective space was equivalent to failure of versality of a certain unfolding. Here we study in detail (reduced) plane curves of degree $d \geq \underline{3}$, excluding the trivial case of cones.

We enumerate all possible group actions - these have to be either semisimple or unipotent - for any degree $d$. A 2-parameter group can only occur if $d=3$. Explicit lists of singularities of the corresponding curves are given in the cases $d \leq 6$. We also show that the projective classification of these curves coincides - except in the case of the group action with weights $[-1,0,1]$ - with the classification of the singular points.

The sum $\tau$ of the Tjurina numbers of the singular points is either $d^{2}-3 d+3$ or $d^{2}-3 d+2$ while, for $d \geq 5$, if there is no group action we have $\tau \leq d^{2}-4 d+7$. We give $\mu=\tau$ in the semi-simple case; in the unipotent case, we determine the values of both $\mu$ and $\tau$.

In the semi-simple case, we show that the unfolding mentioned above is also topologically versal if $d \geq 6$; in the unipotent case this holds at least if $d=6$.
\end{abstract}

\section{Background}

Throughout this section, $\Gamma$ will be a reduced curve of degree $d \geq \underline{3}$, with homogeneous equation $f=0$.

We will say that $\Gamma$ has $k$-dimensional symmetry if it admits a

1991 Mathematics Subject Classification: 14B05, 14 N05.

Servicio Publicaciones Univ. Complutense. Madrid, 1999. 
k-dimensional algebraic subgroup of $P G L_{2}(C)$ as automorphism group.

We will say that $\Gamma$ is $a$-versal if adding homogeneous functions of degree $2(d-2)-1-a$ to $f$ induces (in an appropriate affine chart) a simultaneous versal deformation of the singularities of $\Gamma$; and that $\Gamma$ is a-non-versal if this fails.

Combining $[3,1.1]$ in the case of curves with $[3,1.6]$ characterises reduced curves with 1-dimensional symmetry:

Proposition 1.1. The following are equivalent:

(1) Г has 1-dimensional symmetry,

(2) There exists a linear vector field which annihilates $f$,

(3) $\Gamma$ is 1-non-versal.

Symmetry of higher dimension is usually not possible:

Proposition 1.2. If $\mathrm{\Gamma}$ has 2-dimensional symmetry, then $d=3$.

Proof. Let $\xi^{\prime}, \eta^{\prime}$ be elements of the Lie algebra of the 2-dimensional automorphism group, and let $\xi, \eta$ be linear vector fields in $C^{3}$ lifting these. Then $\xi, \eta$ annihilate $f$, so their cross-product is parallel to the gradient $\nabla f$.

Thus, since the cross-product has components of degree 2, whilst $\nabla(f)$ has components of degree $d-1$, the cross-product can only be non-zero if $d-1 \leq 2$; so $\xi, \eta$ can only be linearly independent if $d=3$.

The next result is a combination of $[6,3.2]$ in case $r=1$ and the arguments used in its proof. We will write $\tau(\Gamma)$ for the sum of the Tjurina numbers of the singularities of $\Gamma$.

\section{Proposition 1.3.}

(1) Stippose that no non-zero linear vector field annihilates $f$.

Then

$$
\tau(\Gamma) \leq\left\{\begin{array}{cc}
1 & \text { if } d=3 \\
6 & \text { if } d=4 \\
d^{2}-4 d+7 & \text { if } d \geq 5
\end{array}\right.
$$


(2) Suppose that $\Gamma$ is not a cone, and that there exists a linear vector field $\xi$ which annihilates $f$. Then there are two possibilities:

(a) There is a vector field $\eta$ of degree $d-2$ annihilating $f$ such that $\xi \times \eta=\nabla f$. Then any vector field annihilating $f$ is a linear combination of $\xi, \eta$ and the Hamiltonian vector fields, and $\tau(\Gamma)=$ $d^{2}-3 d+3$.

(b) Any vector field annihilating $f$ is a linear combination of $\xi$ and the Hamiltonian vector fields. Then $\tau(\Gamma)=d^{2}-3 d+2$.

Corollary 1.4. Suppose that $\Gamma$ is not a cone.

(1) If $\Gamma$ has 1-dimensional symmetry, then $\tau(\Gamma)=d^{2}-3 d+3$ or $d^{2}-3 d+2$. Conversely, if $\tau(\Gamma)=d^{2}-3 d+3$, or if $\tau(\Gamma)=d^{2}-3 d+2$ and $d \neq 4,5$, then $\Gamma$ has 1 -dimensional symmetry.

(2) $\Gamma$ has 2-dimensional symmetry if and only if $d=3$ and $\tau(\Gamma)=3$.

Proof. (1) follows at once from 1.1 and 1.3 ; (2) follows from these together with 1.2 and its proof.

\section{Enumeration.}

A 1-dimensional algebraic subgroup of $G L_{n+1}(C)$ is either semisimple or unipotent.

In the semisimple case we can choose coordinates to make the matrix of the infinitesimal generator $\xi=\sum_{0 \leq i, j \leq n} \alpha_{i, j} x_{i} \partial / \partial x_{j}$ diagonal, say $\alpha_{i, j}=c_{i} \delta_{i, j}$, so that the action is $t .\left(x_{0}, \ldots, x_{k}\right)=\left(t^{c_{0}} x_{0}, \ldots, t^{c_{k}} x_{k}\right)$.

Notice that this implies that $c_{0}, \ldots, c_{n}$ are integers. We will denote this group by the symbol $\left[c_{0}, \ldots, c_{k}\right]$.

In the unipotent case, $\xi$ is nilpotent and we may take its matrix in Jordan normal form: a direct sum of Jordan blocks of sizes $b_{1}, \ldots, b_{r}$, where a Jordan block of size $b$ corresponds to the vector field $\sum_{i=1}^{b-1} x_{i} \partial / \partial x_{i+1}$. We write $N i l_{b_{1}, \ldots, b_{r}}$ for the group so defined.

We now enumerate reduced curves $\Gamma$ admitting a 1-dimensional algebraic group $H^{\prime}$ of projective automorphisms. The calculations are very close to those for the case of quintic curves, which was discussed in [8]. 
Let $f(x, y, z)=0$ be an equation of $\Gamma$. Then $H^{\prime}$ lifts uniquely to an algebraic 1-parameter subgroup $H$ of $G L_{3}(C)$, not contained in the centre, and preserving $f$, not just $\Gamma$.

We consider first the case when $H$ is semisimple; we may take it as $\left[w_{1}, w_{2}, w_{3}\right]$. Thus the exponents $(r, s, t)$ for which the coefficient of $x^{r} y^{s} z^{t}$ in $f$ is nonzero satisfy $w_{1} r+w_{2} s+w_{3} t=0$ as well as $r+s+t=d$. Notice that $w_{1}, w_{2}, w_{3}$ are only determined up to a common factor.

We plot the exponents $(r, s, t)$ with $r+s+t=d$ in a triangular lattice, where the $k^{\text {th }}$ row consists of the $k+1$ entries with $r=d-k$, and with $s$ decreasing along the row. Since the exponents occurring in $f$ satisfy a linear relation, they lie on a line; let $L$ be the least line segment containing them all. Since $\Gamma$ is reduced, for each edge of the triangle at least one end of $L$ must be at distance 0 or 1 from the edge: for if (e.g.) $L$ contains no point from the two lower rows, $f$ is divisible by $x^{2}$. Hence one of the ends of $L$ must be within distance 1 from each of 2 edges, so may be taken as $A=(0, d, 0), B=(1, d-1,0)$ or $C=(1, d-2,1)$. The other end of $L$ must be within 1 of the opposite edge, so may be taken as $\alpha_{r}=(r, 0, d-r)$ or $\beta_{r}=(r, 1, d-r-1)$ for some $r$.

Eliminating repetitions arising from symmetries of the triangle gives the following $4 d-5$ line segments:

$$
\begin{aligned}
A \alpha_{r}\left(0 \leq r \leq \frac{1}{2} d\right), & A \beta_{r}\left(0 \leq r \leq \frac{1}{2}(d-1)\right), \\
B \alpha_{r}(1 \leq r \leq d-1), & B \beta_{r}(1 \leq r \leq d-2) \\
C \alpha_{r}\left(2 \leq r \leq \frac{1}{2} d\right), & C \beta_{r}\left(2 \leq r \leq \frac{1}{2}(d-1)\right) .
\end{aligned}
$$

The cases $A \alpha_{0}, A \beta_{0}$, where the curve is just a cone on $d$ points, are more degenerate than the others; here an equation is annihilated by a vector field of degree 0 . The weights can be taken to be $[-1,0,0]$. There is, of course, just one singularity, with $\mu=\tau=(d-1)^{2}$. We will not consider these cases any further.

The only other cases where the line segment is parallel to a side of the triangle, so that two weights are equal, are $B \alpha_{1}, B \beta_{1}$, where the curve is a cone on $d-1$ points plus a line; here the weights can be taken to be $[-(d-1), 1,1]$. The cone singularity has $\mu=\tau=(d-2)^{2}$; and there are $d-1$ further $A_{1}$ singularities where the extra line meets the cone. We note that the projective classification of such curves corresponds exactly 
to the $\mathcal{K}$-classification of the cone singularities, and hence the multi- $\mathcal{K}$ classification of the constellation of singularities consisting of a cone of degree $d-1$ and $d-1 A_{1}$ 's.

There are $d-4$ moduli for this classification.

In all other cases, the weights are distinct, and the choice of representative line segments made above ensures that 0 and $w_{2}$ lie between $w_{1}$ and $w_{3}$. We choose signs so that $w_{1}<w_{2}<w_{3}$ and $w_{1}<0<w_{3}$; this is achieved by taking $\left(w_{1}, w_{2}, w_{3}\right)$ as the negative of the cross-product of the coefficients of the given end-points, at least up to a positive factor.

The weights distinguish the line segments except in the cases noted above where two weights are equal, and in the cases $A \alpha_{k}, C \alpha_{k}(d=2 k)$ and $A \beta_{k}, C \beta_{k}(k>1, d=2 k+1)$, where the weights may be taken as $[-1,0,1]$.

When the weights are distinct, the only possibilities for singularities are the unit points $[(1,0,0)],[(0,1,0)]$ and $[(0,0,1)]$; for the vector $\left(w_{1} x, w_{2} y, w_{3} z\right)$ is only parallel to the kernel (spanned by $(x, y, z)$ at $(x, y, z))$ of the tangent map to the projection $C^{3} \backslash 0$ to $C P^{2}$ above these points. Indeed, examination of equations shows that $[(0,1,0)]$ does not even lie on the curves corresponding to line segments with end-point $A$ (where the equation has non-zero coefficient for $y^{d}$ ), is a non-singular point of those with end-point $B$, and is an $A_{1}$ singularity of those with end-point $C$; whilst there is a singularity at $[(1,0,0)]$ except in the case $B \alpha_{d-1}$, and there is a singularity at $[(0,0,1)]$ except in the case $A \alpha_{1}$.

All these singularities are in fact weighted-homogeneous. For, using the Euler relation $d f=x \partial f / \partial x+y \partial f / \partial y+z \partial f / \partial z$, we find

$$
\begin{aligned}
& 0=w_{1} x \partial f / \partial x+w_{2} y \partial f / \partial y+w_{3} z \partial f / \partial z \\
& =-\left\{\left(-w_{1} d\right) f-\left(w_{2}-w_{1}\right) y \partial f / \partial y-\left(w_{3}-w_{1}\right) z \partial f / \partial z\right\}
\end{aligned}
$$

so that the weights at $[(1,0,0)]$ are $\left(w_{2}-w_{1}, w_{3}-w_{1}\right) /-d w_{1}$. Similarly, the weights at $[0,1,0)],[(0,0,1)]$ are $\left(w_{2}-w_{1}, w_{2}-w_{3}\right) / d w_{2},\left(w_{3}-\right.$ $\left.w_{1}, w_{3}-w_{2}\right) / d w_{3}$, respectively.

When the weights are distinct, the connected component of the identity in the subgroup of $G L_{3}(C)$ which preserves the weights consists of the diagonal matrices alone, corresponding to scaling the variables. It follows that the projective classification of the curves with these weights has $N-2$ moduli, where $N$ is the number of lattice points on the corresponding line segment $L$ - an appropriate normal form for the corre- 
sponding equations has coefficients 1 for the monomials corresponding to the end-points of $L$, whilst the coefficients of the other monomials give the moduli.

As to the multi- $\mathcal{K}$-classification of the singular points, we have:

Proposition 2.1. Let $L$ be one of the line segments of (1).

If $d=2 k$ and $L=A \alpha_{k}$, or if $d=2 k+1$ and $L=A \beta_{k}$, then, for each possible multi-K-class, there is a 1-parameter family of projectively inequivalent curves with line segment $L$ whose singular points have this class.

In all other cases the multi-K-classification of the singular points of curves with line segment $L$ coincides with the projective classification of the curves.

Proof. We may restrict attention to curves with the equation implied by the corresponding line segment.

For the first statement, note that the equation is of form $p(x z, y)$, where $p$ is a polynomial in two variables weighted-homogeneous with respect to $(2,1) / d$. The curves with equations $p\left(x z+t y^{2}, y\right)$ also have, except for one value of $t$ where the coefficient of $y^{d}$ vanishes, line segment $L$, and are projectively distinct for distinct $t \in C$. On the other hand, the singularities of these curves at $[(1,0,0)]$ and $[(0,0,1)]$ have equations $p\left(z+t y^{2}, y\right)$ and $p\left(x+t y^{2}, y\right)$, so have constant $\mathcal{K}$-class for all $t \in C$. The statement is proved.

The second statement was shown earlier in the cases where two weights are equal; so we suppose that the weights are distinct. Since $\mathcal{K}$-equivalence of weighted homogeneous functions is the same as equivalence with respect to weight-preserving coordinate change, the statement fails for a given $L$ only if the weights at both $[(1,0,0)]$ and $[(0,0,1)]$ admit weight-preserving diffeomorphisms other than simple scaling. This requires that the weights be $(1, k)$ and $(\ell, 1)$ at these points, with $k, \ell$ positive integers. Thus $\frac{1}{k}\left(w_{3}-w_{1}\right)=w_{3}-w_{2}$ and $\frac{1}{\ell}\left(w_{3}-w_{1}\right)=w_{2}-w_{1}$. Adding, we find $\frac{1}{k}+\frac{1}{\ell}=1$, so $k=\ell=2$. Moreover, for the statement to fail for $L$, applying the transformation $z \rightarrow z+t y^{2}$ to $\left.m\right|_{x=1}$, where $m$ is a monomial represented on $L$, must yield, on homogenizing to degree $d$ with $x$, a $C$-linear combination of monomials represented on $L$. Thus, if $a_{3}$ is the greatest power of $z$ occurring in a monomial represented on $L$, then there must exist $a_{1}, a_{2} \geq 0$ such that $x^{a_{1}} y^{2 a_{3}+a_{2}}$ is represented on $L$; so $a_{1} w_{1}+\left(2 a_{3}+a_{2}\right) w_{2}=0$. Similarly, working at $[(0,0,1)]$, there 
exist $b_{1}>0, b_{2}, b_{3} \geq 0$ such that $\left(2 b_{1}+b_{2}\right) w_{2}+b_{3} w_{3}=0$. Since $w_{1}, w_{3}$ have opposite signs, we conclude that $a_{2}=b_{2}=0$, and so $w_{2}=0$. Hence $w_{1}=-w_{3}$, and, since $\left(0,2 a_{3}+a_{2}, 0\right) \in L, L$ is $A \alpha_{k}$ if $d=2 k$, and $A \beta_{k}$ if $d=2 k+1$. The proof is complete.

We turn to the case when $H$ is unipotent. It is then conjugate to one of the subgroups

$$
\begin{aligned}
& N_{i l_{1,2}}: t .(x, y, z)=(x, y, z+t y), \\
& N i l_{3}: t .(x, y, z)=\left(x, y+t x, z+t y+\frac{1}{2} t^{2} x\right) .
\end{aligned}
$$

Now a $N i l_{1,2}$-invariant function is independent of $z$, so in this case $\Gamma$ is a cone. We will not consider this case further.

For $\mathrm{Nil}_{3}$ the ring of invariant polynomials is generated by $x$ and $y^{2}-2 x z$. Thus a reduced $N i l_{3}$-invariant function of degree $d=2 k$ is a scalar multiple of a product $\prod_{i=1}^{k}\left(y^{2}-2 x z+4 a_{i} x^{2}\right)$, with the $a_{i}$ all distinct; whilst if $d=2 k+1$ it is the product of such a function by $x$. The projective classification thus has $k-2$ moduli.

Geometrically, the curve is, if $d=2 k$, the union of $k$ smooth conics meeting in just one point, or, if $d=2 k+1$, the union of $k$ smooth conics and a line which meet in just one point. In each case, then, there is just one singularity, at $[(0,0,1)]$.

This singularity is not quasi-homogeneous for $d \geq \underline{5}$, though this is quite difficult to see directly. It will follow from the results of 3.1 , which show that the singularity's Milnor and Tjurina numbers are not equal when $d \geq 5$.

Proposition 2.2. The projective classification of curves with unipotent symmetry coincides with the $\mathcal{K}$-classification of the singular point.

Proof. Apart from scaling, the only projective transformations preserving the normal form given above are of form $z \rightarrow z+\alpha x$. Thus we can arrange that $a_{1}=0, a_{2}=1$, whilst the remaining $a_{i}$ are moduli for the projective classification.

For the $\mathcal{K}$-classification, we work in the affine chart $z=1$, and use the local coordinate system $X=y^{2}-2 x z, y$. Thus, writing $g=\left.f\right|_{z=1}$, we have

$$
g(X, y)=\left\{\begin{array}{cl}
\prod_{i=1}^{k}\left(X+a_{i}\left(X-y^{2}\right)^{2}\right) & \text { if } d=2 k \\
\left(X-y^{2}\right) \prod_{i=1}^{k}\left(X+a_{i}\left(X-y^{2}\right)^{2}\right) & \text { if } d=2 k+1
\end{array}\right.
$$


We assign weights 4,1 to $X, y$ respectively. Then the lowest-weight terms in $g$ are

$$
\bar{g}(X, y)=\left\{\begin{array}{cl}
\prod_{i=1}^{k}\left(X+a_{i} y^{4}\right) & \text { if } d=2 k \\
-y^{2} \prod_{i=1}^{k}\left(X+a_{i} y^{4}\right) & \text { if } d=2 k+1 .
\end{array}\right.
$$

Now let $h=\left(h_{1}, h_{2}\right)$ be a germ of diffeomorphism of $\left(C^{2},(0,0)\right)$, and write $\bar{h}=\left(\bar{h}_{1}, \bar{h}_{2}\right)$ for its terms of least weight. Then the terms of least weight of $g \circ h$ are $\bar{h}_{1} \partial \bar{g} / \partial X$, or $\bar{h}_{2} \partial \bar{g} / \partial y$, or their sum if these have equal weight. If $h$ is the source component of a $\mathcal{K}$-transformation preserving the normal form of $g$, we see that $\bar{h}_{1}$ must be of weight $4, \bar{h}_{2}$ of weight 1 , and that $\bar{h}$ preserves the normal form of $\bar{g}$ up to multiplication by a non-zero constant. We conclude that, apart from scaling, the only transformations preserving $\bar{g}$ are of form $X \rightarrow X+\alpha y^{4}$. So we can arrange that $a_{1}=0, a_{2}=1$, whilst the remaining $a_{i}$ are moduli for the $\mathcal{K}$-classification. Thus the $\mathcal{K}$-classification is at least as fine as the projective classification. Since it cannot be finer, the proof is complete.

We conclude this section by giving tables with explicit lists for the cases $d=3,4,5,6$. Our notation for singularities in general follows that of Arnold, with some modifications; the notation for trimodal singularities is introduced in [9].

The tables are arranged in order given by the nature of the singularities, and a horizontal rule is placed at a point in the table where the modality increases; we list the semi-simple cases first and then the unipotent cases.

In the equations, $a_{r}\left(v_{1}, v_{2}\right)$ denotes a homogeneous polynomial of degree $r$ in $v_{1}, v_{2}$. For the corresponding curve to be reduced, the equation must have no repeated factors; so the polynomial $a_{r}$ must have distinct roots, and in some cases one or both of the coefficients of $v_{1}^{r}, v_{2}^{r}$ must also be non-zero.

We also give the sums $\mu$ and $\tau$ of the Milnor and Tjurina numbers of the singularities which appear.

Cubic curves with 1- and 2-dimensional symmetry

\begin{tabular}{ccccc} 
Group & Segment & Equation & Singularities & $\tau=\mu$ \\
\hline$[-1,0,1]$ & $A \beta_{1}$ & $y^{3}+x y z$ & $2 A_{1}$ & 2 \\
{$[-2,0,1]$} & $A \alpha_{1}$ & $y^{3}+x z^{2}$ & $A_{2}$ & 2 \\
{$[-1,0,1] \cdot[0,-1,1]$} & $B \alpha_{1}, B \beta_{1}$ & $x y z$ & $3 A_{1}$ & 3 \\
{$[-2,1,4] . N i_{3}$} & $B \alpha_{2}$ & $x\left(y^{2}-2 x z\right)$ & $A_{3}$ & 3
\end{tabular}


All cubic curves with $\tau=2$ have 1-dimensional symmetry, whilst those with $\tau=3$ have 2-dimensional symmetry. To see the second statement directly, observe that curves corresponding to the segments $B \alpha_{1}, B \beta_{1}$ are projectively equivalent to $x y z$, whilst curves corresponding to the segment $B \alpha_{2}$ are projectively equivalent to $x\left(y^{2}-2 x z\right)$. These types are all projectively unique.

Quartic curves with 1-dimensional symmetry

\begin{tabular}{ccccc} 
Group & Segment & Equation & Singularities & $\tau=\mu$ \\
\hline$[-1,0,1]$ & $A \alpha_{2}$ & $a_{2}\left(x z, y^{2}\right)$ & $2 A_{3}$ & 6 \\
{$[-1,0,1]$} & $C \alpha_{2}$ & $x y^{2} z+x^{2} z^{2}$ & $2 A_{3}+A_{1}$ & 7 \\
{$[-3,1,3]$} & $B \alpha_{2}$ & $x^{2} z^{2}+x y^{3}$ & $A_{5}+A_{2}$ & 7 \\
{$[-3,1,1]$} & $B \alpha_{1}, B \beta_{1}$ & $x a_{3}(y, z)$ & $D_{4}+3 A_{1}$ & 7 \\
{$[-2,0,1]$} & $A \beta_{1}$ & $y^{4}+x y z^{2}$ & $D_{5}+A_{1}$ & 6 \\
{$[-3,1,5]$} & $B \beta_{2}$ & $x y^{3}+x^{2} y z$ & $D_{6}+A_{1}$ & 7 \\
{$[-3,0,1]$} & $A \alpha_{1}$ & $y^{4}+x z^{3}$ & $E_{6}$ & 6 \\
{$[-3,1,9]$} & $B \alpha_{3}$ & $x y^{3}+x^{3} z$ & $E_{7}$ & 7 \\
\hline$N i l_{3}$ & & $a_{2}\left(x^{2}, y^{2}-2 x z\right)$ & $A_{7}$ & 7
\end{tabular}

The classification arises in the theory of del Pezzo surfaces: for (see [2, p.67]) the anticanonical model of a del Pezzo surface of degree two is a double plane branched along a quartic curve with simple singularities.

All quartic curves with $\tau=7$ have 1-dimensional symmetry; as do those with singularities $2 A_{3}$. There are two $P G L_{2}(C)$-orbits of curves with singularities $D_{5}+A_{1}$ (a cuspidal cubic and cuspidal chord), one without 1-dimensional symmetry and one with (when the chord passes through the flex). Similarly, there are two $P G L_{2}(C)$-orbits of curves with an $E_{6}$ singularity; for the curves with 1-dimensional symmetry, the two flexes coalesce to a hyperflex.

There is a 1-parameter family of projectively inequivalent symmetric curves with singularities $2 A_{3}$; all the other types are projectively unique. 
Quintic curves with 1-dimensional symmetry

\begin{tabular}{cccccc} 
Group & Segment & Equation & Singularities & $\tau$ & $\mu$ \\
\hline$[-1,0,1]$ & $A \beta_{2}$ & $y a_{2}\left(x z, y^{2}\right)$ & $2 D_{6}$ & 12 & 12 \\
{$[-1,0,1]$} & $C \beta_{2}$ & $x y^{3} z+x^{2} y z^{2}$ & $2 D_{6}+A_{1}$ & 13 & 13 \\
{$[-8,2,7]$} & $B \beta_{2}$ & $x y^{4}+x^{2} y z^{2}$ & $D_{8}+D_{5}$ & 13 & 13 \\
{$[-12,3,8]$} & $B \alpha_{2}$ & $x y^{4}+x^{2} z^{3}$ & $A_{7}+E_{6}$ & 13 & 13 \\
{$[-9,1,6]$} & $C \alpha_{2}$ & $x y^{3} z+x^{2} z^{3}$ & $E_{7}+A_{5}+A_{1}$ & 13 & 13 \\
{$[-3,0,2]$} & $A \alpha_{2}$ & $y^{5}+x^{2} z^{3}$ & $E_{8}+A_{4}$ & 12 & 12 \\
\hline$[-4,1,1]$ & $B \alpha_{1}, B \beta_{1}$ & $x a_{4}(y, z)$ & $T_{2,4,4}+4 A_{1}$ & 13 & 13 \\
{$[-4,1,6]$} & $B \alpha_{3}$ & $x a_{2}\left(y^{2}, x z\right)$ & $T_{2,3,6}+A_{3}$ & 13 & 13 \\
{$[-3,0,1]$} & $A \beta_{1}$ & $y^{5}+x y z^{3}$ & $Z_{11}+A_{1}$ & 12 & 12 \\
{$[-4,1,1]$} & $B \beta_{3}$ & $x y^{4}+x^{3} y z$ & $Z_{12}+A_{1}$ & 13 & 13 \\
{$[-4,0,1]$} & $A \alpha_{1}$ & $y^{5}+x z^{4}$ & $W_{12}$ & 12 & 12 \\
{$[-4,1,16]$} & $B \alpha_{4}$ & $x y^{4}+x^{4} z$ & $W_{13}$ & 13 & 13 \\
\hline$N i l_{3}$ & & $x a_{2}\left(x^{2}, y^{2}-2 x z\right)$ & $T_{2,3,10}$ & 13 & 14
\end{tabular}

Our interest in quintic curves arose from the study of the stratification of singularities in the $\mu$-constant stratum denoted $N_{16}$. The list above was already obtained in [8].

All quintic curves with $\tau=13$ have 1-dimensional symmetry; as do those with singularities $2 D_{6}$. There are two $P G L_{2}(C)$-orbits of curves with each of the singularity constellations $E_{8}+A_{4}, Z_{11}(g h)+A_{1}$ and $W_{12}(q h)$ (here $q h$ signifies the quasi-homogeneous $\mathcal{K}$-class).

One of these orbits contains curves with 1-dimensional symmetry and one curves without. Normal forms, taken from [8], for those without 1-dimensional symmetry are

$$
\begin{array}{ll}
y^{5}+\left(y^{2}+x z\right)^{2} z & \left(E_{8}+A_{4}\right) \\
y^{5}+x y z^{3}+z^{2} y^{3} & \left(Z_{11}(q h)+A_{1}\right) \\
y^{5}+x z^{4}+z^{3} y^{2} & \left(W_{12}(q h)\right)
\end{array}
$$

There are 1-parameter families of projectively inequivalent symmetric curves with singularities $2 D_{6}, T_{2,4,4}+4 A_{1}$ and $T_{2,3,6}+A_{3}$; all the other types are projectively unique. The curves with singularities of given multi- $\mathcal{K}$-class in $T_{2,4,4}+4 A_{1}$ or $T_{2,3,6}+A_{3}$ form a single $P G L_{2}(C)$-orbit. 
Sextic curves with 1-dimensional symmetry

\begin{tabular}{|c|c|c|c|c|c|}
\hline Group & Segment & Equation & Singularities & $\tau$ & $\mu$ \\
\hline$[-5,1,5]$ & $B \alpha_{3}$ & $x y^{5}+x^{3} z^{3}$ & $E_{13}+E_{8}$ & 21 & 21 \\
\hline$[-5,1,3]$ & $B \beta_{2}$ & $x y^{5}+x^{2} y z^{3}$ & $Z_{11}+D_{10}$ & 21 & 21 \\
\hline$[-11,1,7]$ & $C \beta_{2}$ & $x y^{4} z+x^{2} y z^{3}$ & $Z_{12}+D_{8}+A_{1}$ & 21 & 21 \\
\hline$[-3,0,2]$ & $A \beta_{2}$ & $y^{6}+x^{2} y z^{3}$ & $Z_{13}+D_{7}$ & 20 & 20 \\
\hline$[-10,2,5]$ & $B \alpha_{2}$ & $x y^{5}+x^{2} z^{4}$ & $W_{12}+A_{9}$ & 21 & 21 \\
\hline$[-8,1,4]$ & $C \alpha_{2}$ & $x y^{4} z+x^{2} z^{4}$ & $W_{13} \pm A_{7} \pm A_{1}$ & 21 & 21 \\
\hline$[-5,1,7]$ & $B \beta_{3}$ & $x y a_{2}\left(y^{2}, x z\right)$ & $Z_{1,0}+D_{6}$ & 21 & 21 \\
\hline$[-2,0,1]$ & $A \alpha_{2}$ & $a_{2}\left(y^{3}, x z^{2}\right)$ & $W_{1,0}+A_{5}$ & 20 & 20 \\
\hline$[-5,1,10]$ & $B \alpha_{4}$ & $x y^{5}+x^{4} z^{2}$ & $W_{17}+A_{4}$ & 21 & 21 \\
\hline$[-5,1,1]$ & $\bar{B} \alpha_{1}, B \beta_{1}$ & $x a_{5}(x, y)$ & $N A_{0,0}+5 A_{1}$ & 21 & 21 \\
\hline$[-4,0,1]$ & $A \beta_{1}$ & $y^{6}+x y z^{4}$ & $N C_{19}+A_{1}$ & 20 & 20 \\
\hline$[-5,1,19]$ & $B \beta_{4}$ & $x y^{5}+x^{4} y z$ & $N C_{20}+A_{1}$ & 21 & 21 \\
\hline$[-5,0,1]$ & $A \alpha_{1}$ & $y^{6}+x z^{5}$ & $N F_{20}$ & 20 & 20 \\
\hline$[-5,1,25]$ & $B \alpha_{5}$ & $x y^{5}+x^{5} z$ & $N F_{21}$ & 21 & 21 \\
\hline$[-1,0,1]$ & $A \alpha_{3}$ & $a_{3}\left(x z, y^{2}\right)$ & $2 T_{2,3,6}$ & 20 & 20 \\
\hline$[-1,0,1]$ & $C \alpha_{3}$ & $x z a_{2}\left(x z, y^{2}\right)$ & $2 T_{2,3,6}+A_{1}$ & 21 & 21 \\
\hline $\mathrm{Nil}_{3}$ & & $a_{3}\left(x^{2}, y^{2}-2 x_{2}\right.$ & $E_{4,0}$ & 21 & 22 \\
\hline
\end{tabular}

All sextic curves with $\tau=20$ or 21 have 1-dimensional symmetry.

Several of these cases describe parametrised families of $P G L_{2}(C)$ orbits. In the case of singularities $2 T_{2,3,6}$ this yields a 1-parameter family of $P G L_{2}(C)$-orbits for each multi- $\mathcal{K}$-class, but in all other cases there is a unique $P G L_{2}(C)$-orbit for each multi-K-class.

\section{Properties}

We begin by computing some invariants of symmetric curves.

Proposition 3.1. Let $\Gamma$ be an $H$-invariant curve which is not a cone; and suppose that the equation $f$ for $\Gamma$ is in one of the forms given in $\$ 2$.

Suppose $H$ is semi-simple. Then $\tau(\Gamma)=\mu(\Gamma)=d^{2}-3 d+3$ and $\chi(\Gamma)=3$ if $y^{d}$ has non-zero coefficient in $f$, whilst otherwise $\tau(\Gamma)=\mu(\Gamma)=d^{2}-3 d+2$ and $\chi(\Gamma)=2$.

Suppose $H$ is nilpotent. Then $\tau(\Gamma)=d^{2}-3 d+3$. If $d=2 k$ then $\mu(\Gamma)=(k-1)(4 k-1)$ and $\chi(\Gamma)=k+1$, whilst if $d=2 k+1$ then $\mu(\Gamma)=k(4 k-1)$ and $\chi(\Gamma)=k+2$. 
Proof. In fact, $\mu(\Gamma)$ can be calculated from $\chi(\Gamma)$, because, by a general property of projective plane curves, $\mu(\Gamma)-\chi(\Gamma)=d^{2}+3 d$. For $\Gamma$ can be deformed to a smooth curve $\tilde{\Gamma}$; we have $\chi(\tilde{\Gamma})=-\left(d^{2}-3 d\right)$, this being the Euler characteristic of any smooth curve in $P^{2}(C)$. Now $\tilde{\Gamma}$ is obtained from $\Gamma$, topologically speaking, by replacing a cone-like neighbourhood of each singular point by the corresponding Milnor fibre. This Milnor fibre has $\chi=1-\mu$, so $\chi(\tilde{\Gamma})=\chi(\Gamma)-\mu(\Gamma)$.

We calculate $\chi$. Consider first the semisimple cases, where the symmetry group is $C^{*}$. Since each non-trivial $C^{*}$-orbit has $\chi=0$, we have $\chi(\Gamma)=\chi(F)$, where $F$ is the set of fixed points of the action contained in $\Gamma$. If all weights are distinct, there are just three such fixed points, the unit points: $\Gamma$ contains $[(1,0,0)]$ and $[(0,0,1)]$ in all cases, and $[(0,1,0)]$ if and only if $y^{d}$ does not appear in $f$. Otherwise the weights are $[-1,-1, d-1]$, the fixed points are $[(0,0,1)]$ and the line $z=0$. These lie in $\Gamma$; so $\chi(\Gamma)=1+2=3$.

Now we turn to the nilpotent cases, where the symmetry group is $C$. We recall from $\$ 2$ that there is only one singular point, at which there are $k$ smooth branches if $d=2 k, k+1$ smooth branches if $d=2 k+1$. Each non-trivial $C$-orbit has $\chi=1$. These orbits are the branches with the common point removed; so that $\chi(\Gamma)$ is 1 plus the number of branches, as claimed.

Finally, we determine $\tau(\Gamma)$. In the semi-simple cases, all the singularities are weighted homogeneous, and thus have the same Milnor and Tjurina numbers, so $\mu(\Gamma)=\tau(\Gamma)$.

For the unipotent case we must work harder. We show that (a) of $1.2,(2)$ applies. We take $\xi$ as the infinitesimal generator $x \partial / \partial y+y \partial / \partial z$ of $\mathrm{Nil}_{3}$. This annihilates $f$, so $x \partial f / \partial y+y \partial f / \partial z=0$. Hence $\partial f / \partial y$ and $\partial f / \partial z$ have a non-zero common factor $K$ of degree $d-2$, with $\partial f / \partial y=K y, \partial f / \partial z=-K x$. Since $\Gamma$ is singular at $[(0,0,1)]$, the partial derivatives of $f$ vanish there, so are contained in the ideal $(x, y)$. Thus we can write $\partial f / \partial x=L x+M y$, with $L, M$ of degree $d-2$, so that $K \partial f / \partial x=K L x+K M y=-L \partial f / \partial z+M \partial f / \partial y$. Thus the vector field $K \partial / \partial x-M \partial / \partial y+L \partial / \partial z$, of degree $d-2$, annihilates $f$; and $\xi \times \eta=\nabla f$. Thus $\tau(\Gamma)=d^{2}-3 d+3$.

Now we turn our attention to topological versality. We will say that a reduced curve $\Gamma \subset P^{2}(C)$ is topologically a-versal if perturbing $f$ by adding homogeneous functions of degree $2(d-2)-1-a$ induces a 
simultaneous topologically versal deformation of the singularities of $\Gamma$.

Proposition 3.2. Let $\Gamma$ be a reduced curve of degree $d \geq 6$ which is not a cone, and with 1-dimensional semi-simple symmetry. Then $\Gamma$ is topologically 1-versal.

Proof. We take the equation for $\Gamma$ to be in one of the forms given in $\S 2,(1)$, and show that one of the singularities of $\Gamma$ is not simple.

In the case where two weights are equal, there is a cone singularity of degree $d-1$ at $[(0,0,1)]$; this is not simple if $d \geq 4$.

Now consider the cases with distinct weights. The singularity at $[(1,0,0)]$ is weighted homogeneous with respect to weights $\left(w_{2}-w_{1}, w_{3}-\right.$ $\left.w_{1}\right) /-d w_{1}$. It is simple if and only if the weight of its Hessian is less than the weight of its equation, that is, if and only if $2\left(-d w_{1}\right)-2\left(w_{2}-w_{1}\right)-2\left(w_{3}-w_{1}\right)<-d w_{1}$, or, simplifying, $(4-d) w_{1}-2 w_{2}-2 w_{3}<0$. Similarly, the singularity at $[(0,0,1)]$, which is weighted homogeneous with respect to weights $\left(w_{3}-w_{1}, w_{3}-w_{2}\right) / d w_{3}$, is simple if and only if $(d-4) w_{3}+2 w_{1}+2 w_{2}<0$. Adding these inequalities gives $(d-6)\left(w_{3}-w_{1}\right)<0$; since $w_{3}>w_{1}$, we see that if $d \geq 6$ then at least one of the singularities at $[(1,0,0)]$ and $[(0,0,1)]$ is not simple.

Next we note that the infinitesimal generator $\xi$ of the group does not vanish at the non-simple singularity. This follows because in all cases $\xi$ is of form $w_{1} x \partial / \partial x+w_{2} y \partial / \partial y+w_{3} z \partial / \partial z$ with $w_{1}, w_{3}$ non-zero; so it does not vanish at either $[(1,0,0)]$ or $[(0,0,1)]$.

It follows that $[3,2.1]$ can be applied; and $\Gamma$ is indeed topologically 1-versal.

We are only able to prove the corresponding result in the unipotent case when $d=6$.

Proposition 3.3. Let $\Gamma$ be a reduced curve of degree 6 which is not a cone, and with 1-dimensional unipotent symmetry. Then $\Gamma$ is topologically 1-versal.

Proof. According to $\S 2$, we may suppose that $\Gamma$ has homogeneous equation $f$ a non-degenerate cubic in $x^{2}$ and $y^{2}-2 x z$ with non-zero $\left(y^{2}-2 x z\right)^{3}$ term. After a coordinate change of form $z \rightarrow z+c x$, we can suppose that $f$ has the form $f(x, y, z)=\left(y^{2}-2 x z\right)^{3}+p\left(y^{2}-2 x z\right)(2 x)^{4}+q(2 x)^{6}$, with $4 p^{3}+27 q^{2} \neq 0$. The singularity of $\Gamma$ is at $[(0,0,1)]$. According to 1.3 , (2), the lift $\xi=x \partial / \partial y+y \partial / \partial z$ to $C^{3}$ of the infinitesimal generator of the symmetry group generates $(\Delta(f) / H(f))_{1}=\Delta(f)_{1}$. 
We set $X=y^{2}-2 x$; then $X, y$ are local analytic coordinates at $(0,0) \in C^{2}$. The germ $g$ of $f(x, y, 1)$ at $(0,0)$ can be written as $g(X, y)=X^{3}+p X\left(y^{2}-X\right)^{4}+q\left(y^{2}-X\right)^{6}$ with respect to these coordinates. We assign weights 4 to $X, 1$ to $y$. Let $h, w$ be the functiongerms at $(0,0)$ which are given by $h(X, y)=X^{3}+p X y^{8}+q y^{12}$, and $w(X, y)=-2 y^{6}\left(2 p X^{2}+3 q X y^{4}\right)$. Then $g-(h+w)$ is a sum of monomials of weight at least 15 .

We recognise $h$ as representing a weighted homogeneous $\mathcal{K}$-class in $E_{4,0}$; and $w$ represents the socle, of weight 14 , of $\mathcal{O} / J(h)$. Since $g$ and $h+w$ differ by terms of weight at least 15 , we have $w \notin J(g)$, $(g, J(g))=(w, J(h))$, and, by the weighted determinacy theorem of [1, 5.2], $g \cong \cong_{\mathcal{K}} h+w$. It follows also, from the second of these statements, that $\tau(g)=\operatorname{dim}_{C}(\mathcal{O} /(g, J(g)))=21$, confirming the result of 3.1 in this case.

Let $v$ be the function-germ at $(0,0)$ given by $v(X, y)=$ $-2 y^{5}\left(2 p X^{2}+3 q X y^{4}\right)$. Then the projection of $v$ generates the part of $\mathcal{O} / J(h)$ of weight 13 , so that $(v, g, J(g))=(v, w, J(h))$ and $\operatorname{dim}_{C}(\mathcal{O} /(v, g, J(g)))=20$. It follows that $v$ represents an element of the socle of $\mathcal{O} /(g, J(g))$. Since the restriction to $z=1$ of the $\partial / \partial z$ coefficient of $\xi$ is $y$, and $y v=w \notin J(g),[3,2.2]$ shows that $v$ projects to a generator for the reduced Kodaira-Spencer map of the deformation of $g$ induced by perturbing $f$ by homogeneous functions of degree 6 . Let $G$ be the corresponding unfolding of $g$.

Let $K$ be the $\mathcal{K}$-invariant manifold (in a suitable high-order jet-space) generated by $\{h+s v+t w: s, t \in C\}$. (In fact, $K$ consists of three $\mathcal{K}$-classes; those of $h, h+w$ and $h+v$, with Tjurina numbers 22, 21 and 20 , respectively). By the weighted determinacy theorem $[1,5.2]$, $g+s v \cong \kappa h+s v$ for $s \neq 0$. Hence the jets of the family $\{g+s v: s \in C\}$ lie in $K$. Thus $[3,2.3]$ shows that $G$ is transverse to $K$.

By the complex avatar of $[4,10.5 .33], K$ is civilised; from this the topological versality of the unfolding $G$ of $g$ follows by the arguments of $[4,9.4]$, thus giving the topological 1 -versality of $\Gamma$, and completing the proof.

Reduced curves of degree $d=3$ and 4 are versally deformed by the family of all curves of degree $d$, e.g. by the results of [3]. This also holds when $d=5$ except at the cones; but for them the deformation is topologically versal (this follows from the case $a=0$ of $[3,2.1]$; it is essentially a 
special case of Wirthmüller's theorem [10]). The results of 3.2 for $d=6$ and 3.3 come close to showing a similar result in the case $d=6$; it remains only to see that the cones are topologically versally deformed in this case. Proving the required civilization will require an improvement to $[4,9.6 .6]$, however, to allow higher-dimensional instability loci to be considered.

\section{References}

[1] Bruce, J.W., A.A. du Plessis and C.T.C. Wall, Determinacy and unipotency, Invent. Math 88 (1987) 521-554.

[2] Demazure, M., H. Pinkham and B. Teissier (eds.), Seminaire sur les singularités des surfaces, Springer LNM 777, Springer Verlag. 1980.

[3] du Plessis, A.A., Versality properties of projective hypersurfaces, preprint, 1998.

[4] du Plessis, A.A. and C.T.C. Wall, The geometry of topological stability, Oxford University Press. 1995.

[5] du Plessis, A.A. and C.T.C. Wall, Versal deformations in spaces of polynomials of fixed weight, Compositio Math., 114 (1998) 113-124.

[6] du Plessis, A.A. and C.T.C. Wall, Application of the theory of the discriminant to highly singular plane curves, Math. Proc. Camb. Phil. Soc., to appear.

[7] Wall, C.T.C., Notes on the classification of singularities, Proc. London Math. Soc. 48 (1984) 461-513.

[8] Wall, C.T.C., Highly singular quintic curves, Math. Proc. Camb. Phil. Soc., 119 (1996) 257-277.

[9] Wall, C.T.C., Notes on trimodal singularities, preprint, available at http://www.mi.aau.dk/ /esn.

[10] Wirthmüller, K., Universell topologisch trivialen Deformationen, doctoral thesis, Universität Regensburg, 1979. 
Matematisk Institut

ny Munkegade

Aarhus Universitet

8000 Aarhus C.

Denmark

e-mail: matadpomi.aau.dk

Department of Pure Mathematics

The University of Liverpool

Liverpool I. 69 3BX

England

e-mail: C.T.C.Walloliverpool ac.uk

Recibido: 9 de Septiembre de 1998 